\title{
Treatment of tuberculosis and optimal dosing schedules
}

\author{
Kwok Chiu Chang, ${ }^{1}$ Chi Chiu Leung, ${ }^{1}$ Jacques Grosset, ${ }^{2}$ Wing Wai Yew ${ }^{3}$
}

- Additional appendices are published online only. To view these files please visit the journal online (http://thorax.bmj. com).

${ }^{1}$ Tuberculosis and Chest Service, Department of Health, Hong Kong, China 2Department of Medicine, Johns Hopkins University School of Medicine, Baltimore,

Maryland, USA

${ }^{3}$ Tuberculosis and Chest Unit, Grantham Hospital, Hong Kong, China

\section{Correspondence to}

Kwok Chiu Chang, Tuberculosis and Chest Service, Department of Health, Wanchai Chest Clinic 1st Floor, Wanchai Polyclinic, 99 Kennedy Road, Wanchai, Hong Kong; kc_chang@dh.gov.hk

Received 5 August 2010 Accepted 4 November 2010 Published Online First 17 December 2010

\section{ABSTRACT}

Intermittent tuberculosis treatment regimens have been developed to facilitate treatment supervision. Their efficacy has been substantiated by clinical trials and tuberculosis control programmes, notwithstanding the lack of head-to-head comparison between daily and intermittent regimens. Recently, there has been opposing evidence from observational studies, pharmacokinetic-pharmacodynamic studies and animal models that intermittent treatment increases the risk of relapse, treatment failure or acquired rifamycin resistance, especially among HIV-infected patients. Systematic reviews have been conflicting. PubMed, Ovid MEDLINE and EMBASE were systematically searched for publications in English to evaluate the evidence about dosing schedules and treatment efficacy. Levels of evidence and grades of recommendation were assigned largely according to clinical evidence with reference to the Scottish Intercollegiate Guidelines Network guideline development handbook. A total of 32 articles were included after excluding 331 ineligible articles, 42 nonanalytical studies, 22 narrative reviews or expert opinions and 44 articles embedded in systematic reviews. These included 9 systematic reviews, 8 controlled studies, 9 pharmacokinetic-pharmacodynamic studies, 5 mouse studies and 1 article about guinea pig experiments. Findings suggest high levels of evidence for using daily dosing schedules, especially during the initial phase in the presence of cavitation, isoniazid resistance and advanced HIV co-infection, to reduce the risk of treatment failure, recurrence and acquired drug resistance including acquired rifamycin resistance. This review justifies the use of daily schedules in standard tuberculosis treatment regimens (particularly in the initial phase), corroborates prevailing understanding of pharmacokinetics-pharmacodynamics and mycobacterial persisters, and supports exploration of rifapentine-containing regimens in higher dosages and frequency.

\section{INTRODUCTION}

Tuberculosis (TB) is an old infectious disease. Despite the availability of chemotherapy against the tubercle bacillus, our battle with this old human enemy is still far from over. With the rather unusual biological characteristics of this pathogen, ${ }^{1}$ the disease shows a distinctive natural history ${ }^{2} 3$ and a very slow response to existing chemotherapeutic agents. ${ }^{1} 45$ Poor treatment adherence, acquired drug resistance, treatment failure and relapse have been encountered since the early days of chemotherapy. ${ }^{6} \mathrm{~A}$ series of landmark trials in
Madras (now Chennai), ${ }^{7}$ Africa, Hong Kong and Singapore helped to establish the currently adopted 6-month standard regimens given under supervision. ${ }^{9}$ These studies laid the foundation for the global comprehensive strategy for TB control known as directly observed treatment, short-course (DOTS), which was promulgated in 1993 by the World Health Organization (WHO) alongside a declaration of $\mathrm{TB}$ as a global emergency. ${ }^{10}$ Despite some recent controversies over the exact role of the act of directly observed treatment (DOT), ${ }^{11-13}$ no alternative method of drug administration has been conclusively shown to offer a similarly high rate of treatment success as that demonstrated by DOTS under functional programme settings. ${ }^{14-16}$

Intermittent drug delivery either throughout the entire 6-month course or only during the continuation phase in the last 4 months has been widely adopted to facilitate treatment supervision on an outpatient basis ever since the introduction of DOTS. ${ }^{17}$ The lower number of treatment visits helps to reduce both operational and patient-related costs, especially if long travelling distances are involved. As intermittent treatment poses lesser interference on usual lifestyles, patients can carry on their regular daily activities and work. This helps to promote access to care and treatment adherence by patients, especially in resource-limited areas or for underprivileged segments of populations.

In vitro demonstration of the post-antibiotic effect (PAE) has provided the scientific basis for intermittent TB treatment in clinical settings by showing that exposure to drugs, especially isoniazid, for a few hours resulted in suppression of mycobacterial growth for several days. ${ }^{18-21}$ For rifampicin and possibly other TB drugs, free peak drug concentration to minimum inhibitory concentration (MIC) ratio best correlates with the PAE and suppression of resistance. ${ }^{21}$ The PAE has also been suggested by animal studies. A series of guinea pig experiments have shown that, when the same total drug amount is given as a single dose or fractionated into multiple doses of different sizes, better efficacy is observed with high doses given at long intervals, especially for rifampicin and ethambutol. ${ }^{22}$ This suggests concentration-dependent activity in the tested drug. Higher doses in murine models also demonstrated longer PAEs with the exception of rifampicin. ${ }^{23}$ PAEs of TB drugs in humans were first demonstrated in non-rifampicin regimens in a randomised controlled clinical trial that compared twice-weekly isoniazid plus streptomycin and daily isoniazid plus para-aminosalicylic acid in the treatment of pulmonary $\mathrm{TB}^{24}{ }^{24}$ and later in clinical trials of 6-month rifampicin- and isoniazid-containing regimens in the 1970s and 1980s. 
Standard 6-month intermittent regimens have been widely used in TB control programmes with good results. ${ }^{25}{ }^{26}$ Although the exact mechanisms are not fully understood, the PAE is thought to be commonly mediated via suppression of bacterial RNA or protein synthesis. $^{27}$

However, a number of recent studies have challenged the orthodoxy of intermittent treatment. In order to facilitate the development of new $\mathrm{TB}$ drugs and regimens for successful implementation in TB control programmes based on DOTS, we conducted a systematic review to examine the seemingly controversial evidence about dosing intermittency and treatment efficacy across different subpopulations of patients. Measures of treatment efficacy included relapse or recurrence, treatment failure, cure, drug resistance and acquired rifamycin resistance.

\section{METHODS}

PubMed, Ovid MEDLINE and EMBASE were systematically searched through 2 June 2010 for publications in English using a search algorithm that combined the following keywords in Medical Subject Headings, titles, abstracts, or journal titles, as appropriate, with the help of Boolean operators ('and', 'or') and wildcards $\left({ }^{*}\right)$ : (i) tuberculosis; (ii) relapse or recurrence; (iii) treatment and failure; (iv) resistance, and rifamycin, rifampin, rifampicin, rifabutin or rifapentine; (v) intermittent, interruption, once-weekly, twice-weekly, biweekly, three-times-weekly, thriceweekly, once a week, twice a week, thrice a week, dosing schedule, or dosing frequency; (vi) systematic review or metaanalysis; (vii) Cochrane Database Systematic Review or Clinical Evidence; (viii) therapy, chemotherapy, treatment, rifamycin, rifampin, rifampicin, rifabutin, or rifapentine; and (ix) pharmacokinetics and pharmacodynamics. The search algorithm in PubMed is shown in appendix 1 in the online supplement. The above literature search was supplemented by a WHO reference. $^{28}$

The literature search included clinical studies, in vitro studies, animal experiments, narrative reviews or expert opinion, with focus on systematic reviews and controlled clinical studies. Only analytical clinical studies that evaluated the relationship between dosing schedules and treatment efficacy of rifamycinbased regimens or non-analytical clinical studies involving rifamycin-based regimens given for at least 6 months were included. Non-analytical studies, expert opinions and narrative reviews were subsequently excluded when a clinical question could be sufficiently addressed by systematic reviews or controlled clinical studies. Levels of evidence and grades of recommendation were assigned largely according to clinical evidence with reference to the Scottish Intercollegiate Guidelines Network guideline development handbook ${ }^{29}$ (see appendix 2 in the online supplement). Studies rated as having high risk of bias were not used for making recommendations. The risk of bias was judged as high when major potential confounders were not adjusted, low in the absence of quality assessment in systematic reviews or blind assessment when outcome was assessed by objective data, and not applicable for non-conclusive findings.

Studies of non-rifampicin regimens, irrelevant articles and those with no specific information about the impact of dosing schedules on treatment efficacy were excluded. Articles already embedded in systematic reviews were also excluded to reduce materials to a manageable size without losing essential information. Data were extracted by the first author (KCC) and checked by the co-authors for accuracy and interpretation. Disagreement was resolved by consensus.

\section{RESULTS}

The search algorithm initially identified 469 articles. A total of 331 articles were excluded for the following reasons: irrelevance $(n=295)$, effect of dosing schedule on treatment efficacy not evaluated $(n=23)$, rifampicin given for less than 6 months $(n=12)$ and update within the same year $(n=1)$. After further excluding 42 non-analytical studies, 22 narrative reviews or expert opinions and 44 articles already included in systematic reviews, and adding two articles ${ }^{22} 30$ from a WHO reference, ${ }^{28}$ a total of 32 articles were included in the current review (figure 1). These publications included nine systematic reviews with or without meta-analysis, eight controlled studies, nine pharmacokinetic-pharmacodynamic (PK-PD) studies, five studies of TB mouse models and one publication about guinea pig experiments. Studies included in the current review can be grouped under five categories: HIV-related TB, HIV-negative TB, $\mathrm{TB}$ with isoniazid resistance, childhood $\mathrm{TB}$, and in vitro studies and animal experiments.

\section{HIV-related TB}

Table 1 shows three studies regarding the impact of dosing intermittency on treatment efficacy in HIV-related TB: one systematic review with meta-analysis and two retrospective cohort analyses. All suggest that intermittent treatment, especially in the initial phase, reduces treatment efficacy as shown

Search through PubMed, Ovid MEDLINE and EMBASE identified 469 articles

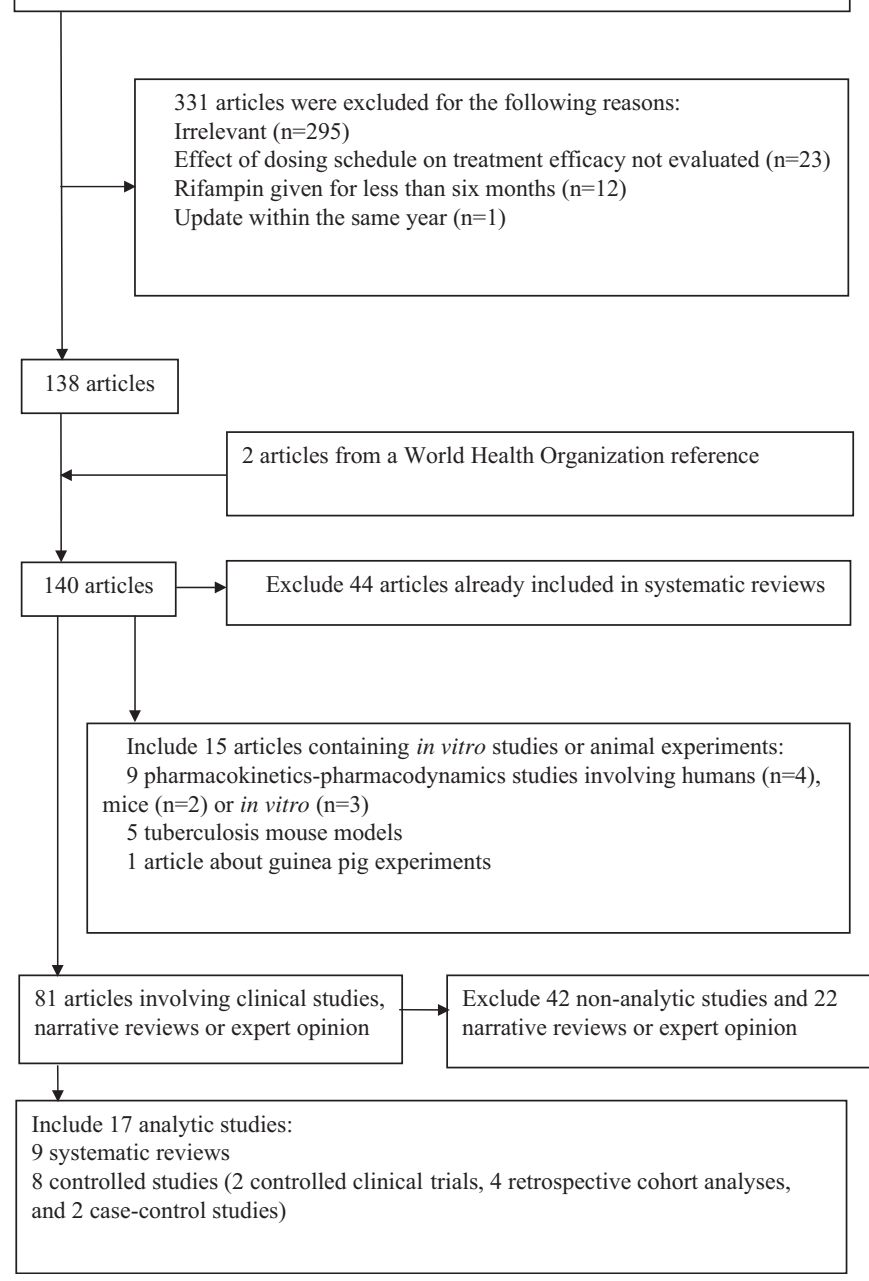

Figure 1 Flow diagram of reviewed articles. 


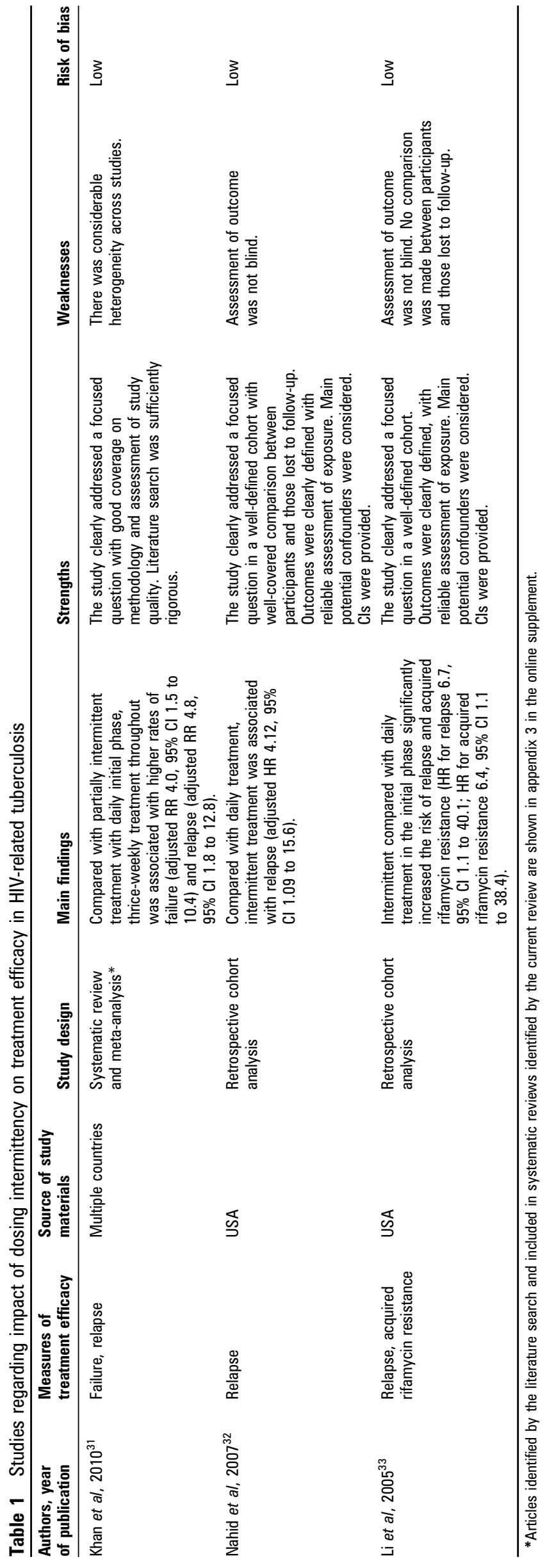

by a higher risk of treatment failure, relapse or acquired rifamycin resistance. The level of evidence is $1+$ and the grade of recommendation for avoiding dosing intermittency, especially in the initial phase in HIV-related TB, is A.

\section{HIV-negative TB}

Table 2 shows 11 studies regarding the impact of dosing intermittency on treatment efficacy in HIV-negative tuberculosis: six systematic reviews with or without meta-analysis, two controlled clinical trials, one retrospective cohort analysis and two case-control studies. The risk of bias was low for four, high for three and not applicable for four. Studies with a low risk of bias suggest that intermittent treatment reduces TB treatment efficacy as shown by a higher risk of relapse or treatment failure. The negative impact appears to be most prominent in the presence of initial cavitation. ${ }^{34}{ }^{35}$ A systematic review of standard 6-month regimens suggests no significant difference between daily treatment throughout and daily treatment in the initial phase. ${ }^{35}$ Among studies with a high risk of bias, all except one suggest that dosing intermittency does not reduce treatment efficacy.

The level of evidence for a negative impact of dosing intermittency on TB treatment efficacy in HIV-negative TB is 1+ and the grade of recommendation for avoiding dosing intermittency, especially in the initial phase in the presence of cavitation, is A.

\section{TB with isoniazid resistance}

Table 3 shows two studies regarding the impact of dosing intermittency on treatment efficacy in TB with isoniazid resistance. Both suggest that dosing intermittency reduces $\mathrm{TB}$ treatment efficacy as shown by a higher risk of treatment failure, relapse or acquired drug resistance. The level of evidence is $1+$ and the grade of recommendation for avoiding dosing intermittency, especially in the initial phase in the presence of isoniazid resistance, is A.

\section{Childhood TB}

Table 4 shows one study regarding the impact of dosing intermittency on treatment efficacy in childhood TB. It suggests that twice-weekly TB treatment may be less efficacious than daily treatment in achieving cure. The level of evidence is $1+$ and the grade of recommendation for avoiding dosing intermittency in childhood TB is A.

\section{In vitro studies and animal experiments}

Table 5 shows 15 in vitro studies and animal experiments. All except two suggest that dosing intermittency may reduce treatment efficacy with internal consistency. PK-PD studies have substantiated the association between dosing intermittency and treatment efficacy by reaffirming that the classical pharmacodynamic parameter, which is the area under the concentrationtime curve (AUC) to MIC ratio, best correlates with the bactericidal or sterilising effect of rifampicin ${ }^{2149}$ and pyrazinamide. ${ }^{50}$ Such findings corroborate the significant association between AUC of rifabutin and failure or relapse in a clinical trial, ${ }^{51}$ the treatment-shortening effect of rifapentine-based regimens in TB mouse models ${ }^{52-54}$ and an increase in the risk of relapse ${ }^{55}$ or treatment failure with acquired resistance to rifampicin or isoniazid following reduction in dosing frequency in $\mathrm{TB}$ mouse models.

\section{DISCUSSION}

The current review suggests high levels of evidence for using daily dosing schedules, especially in the initial phase, to reduce 


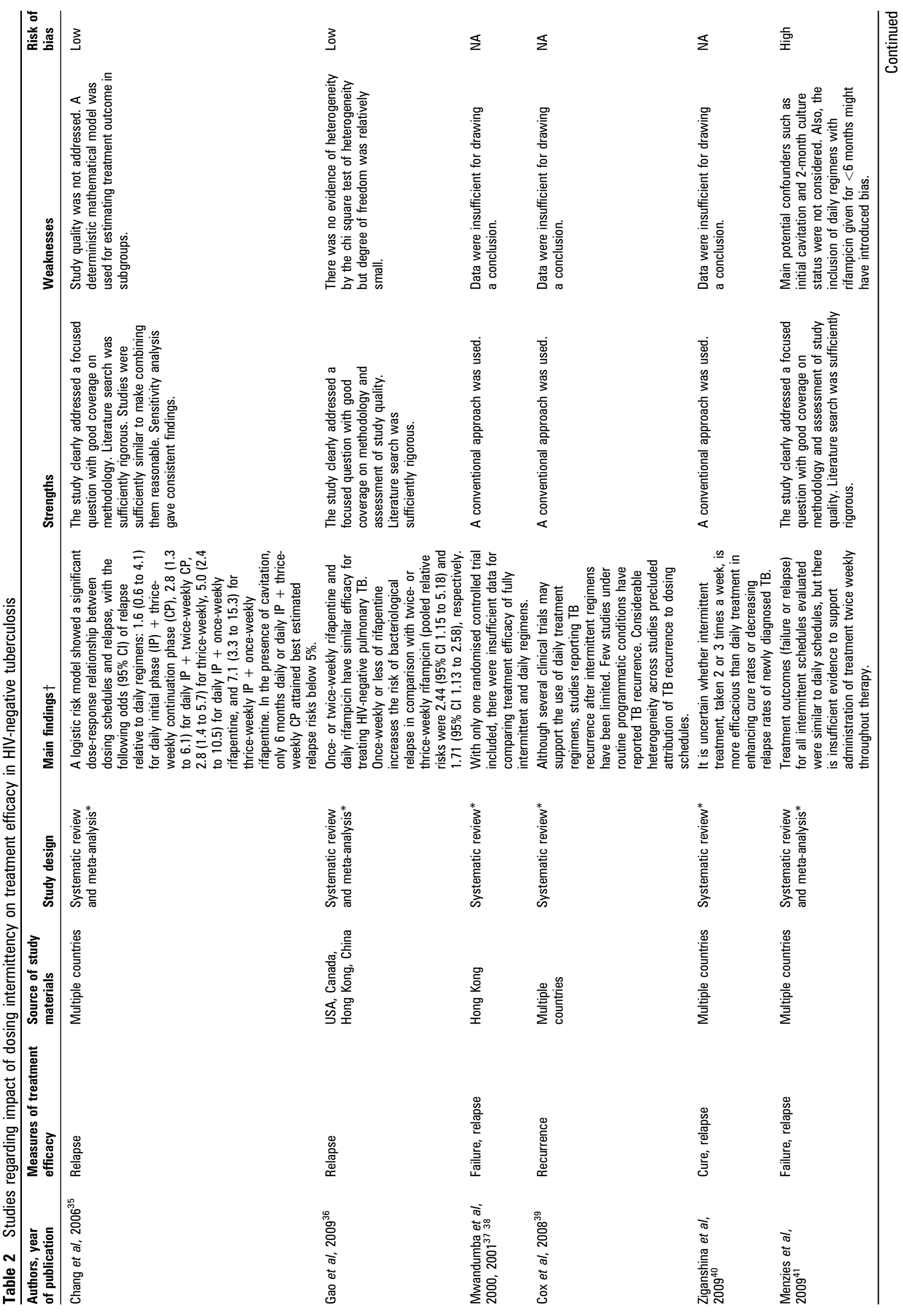




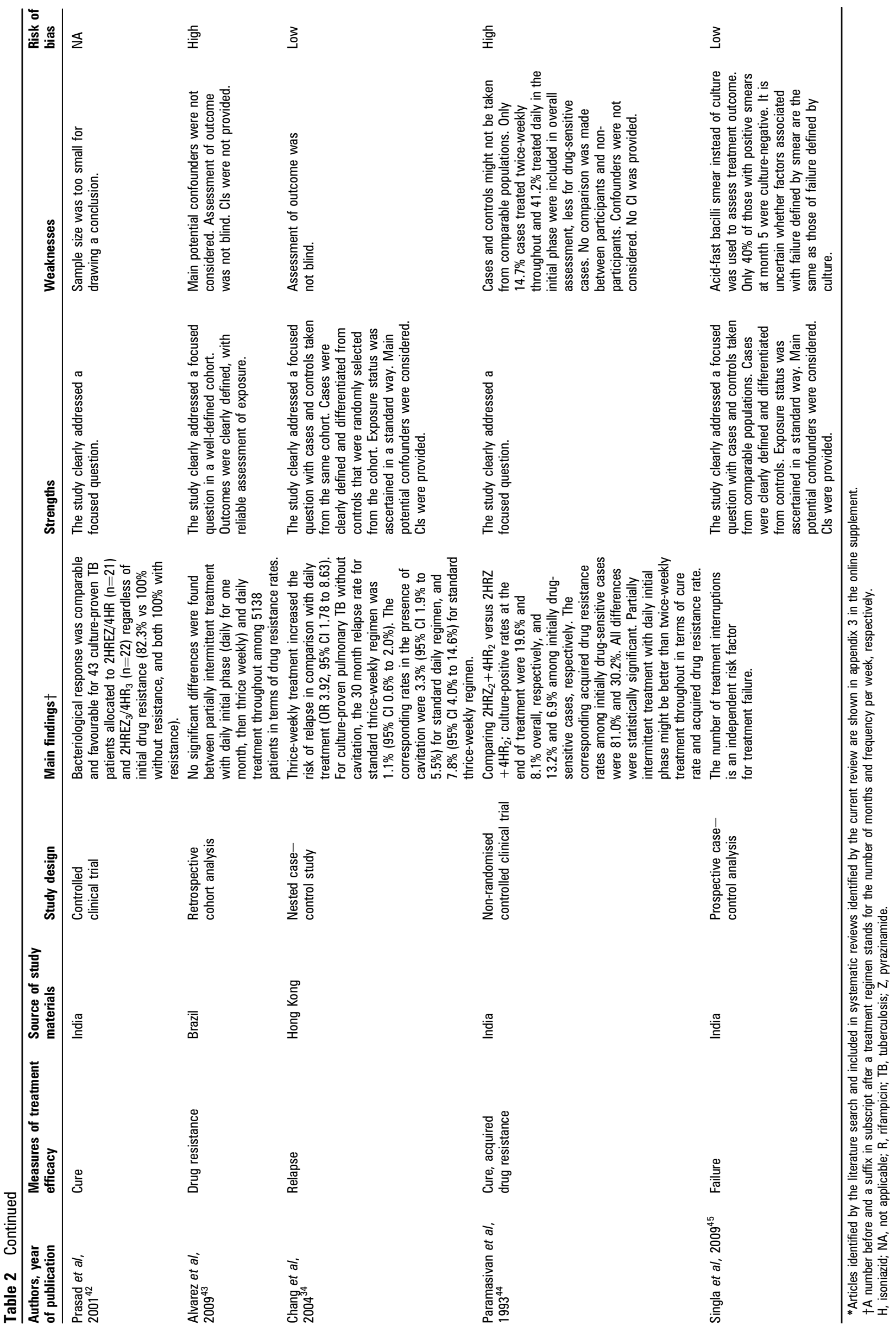



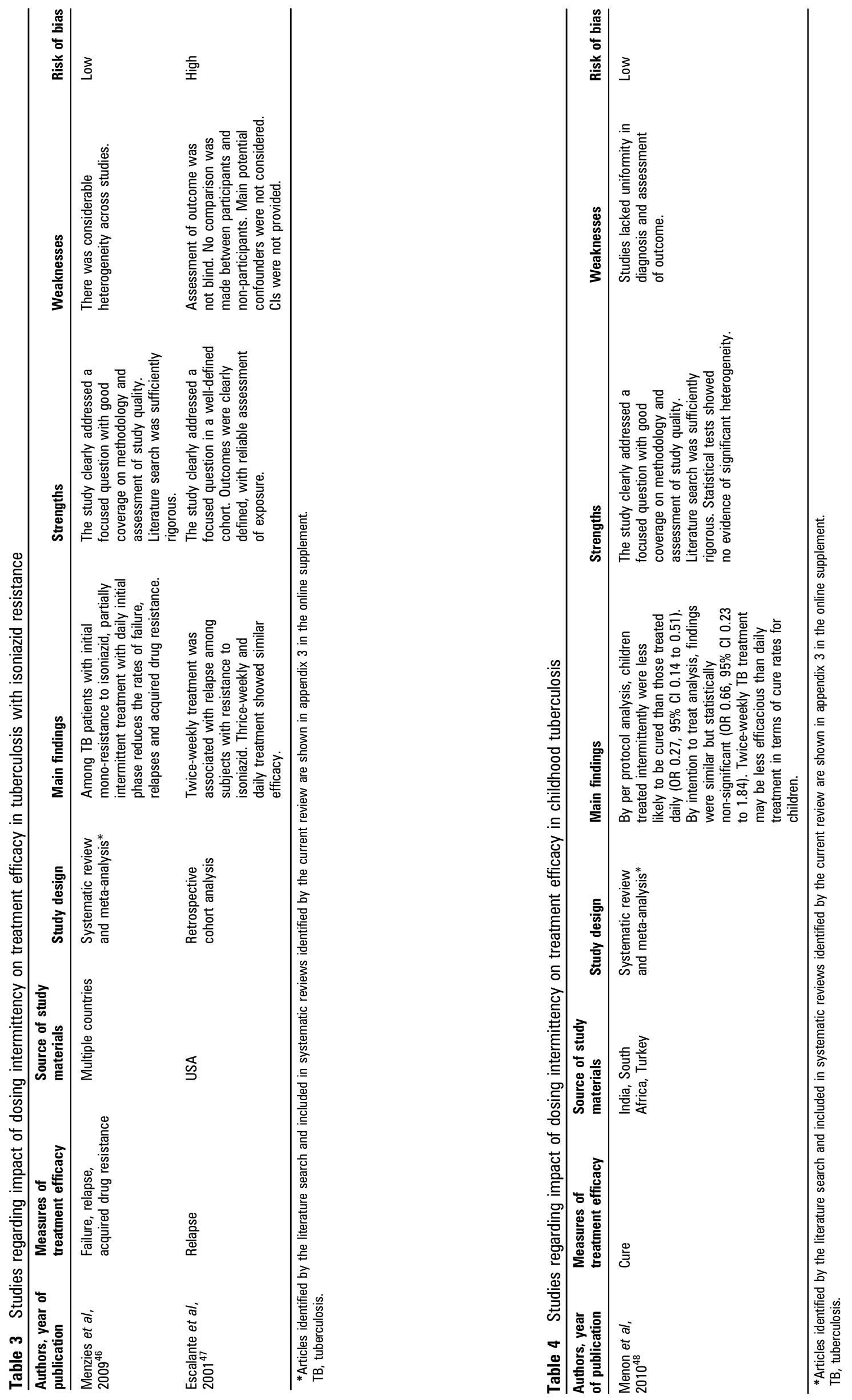

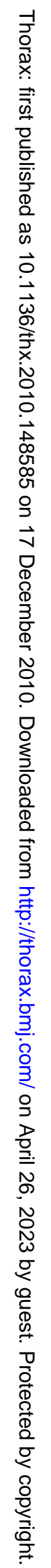




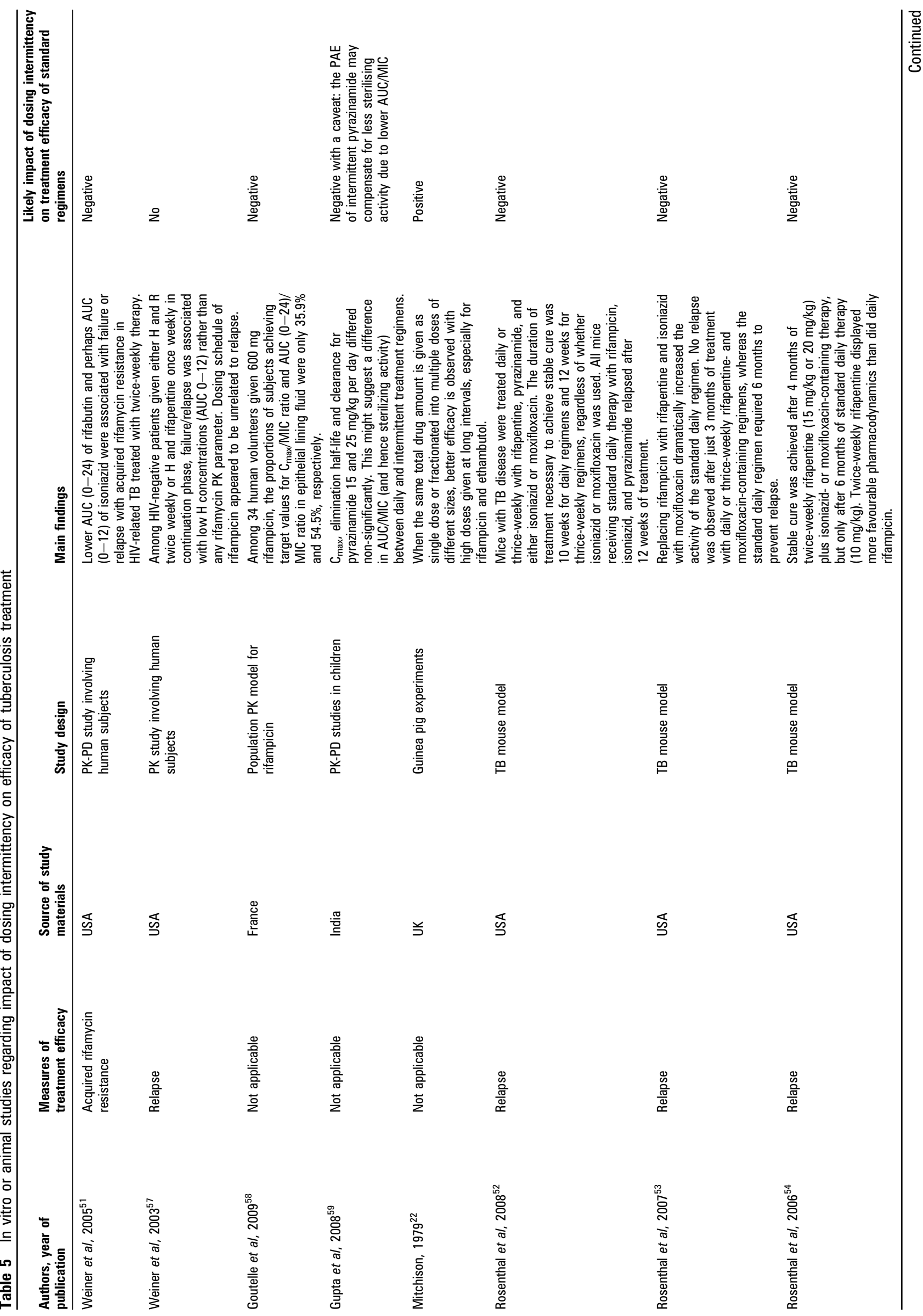




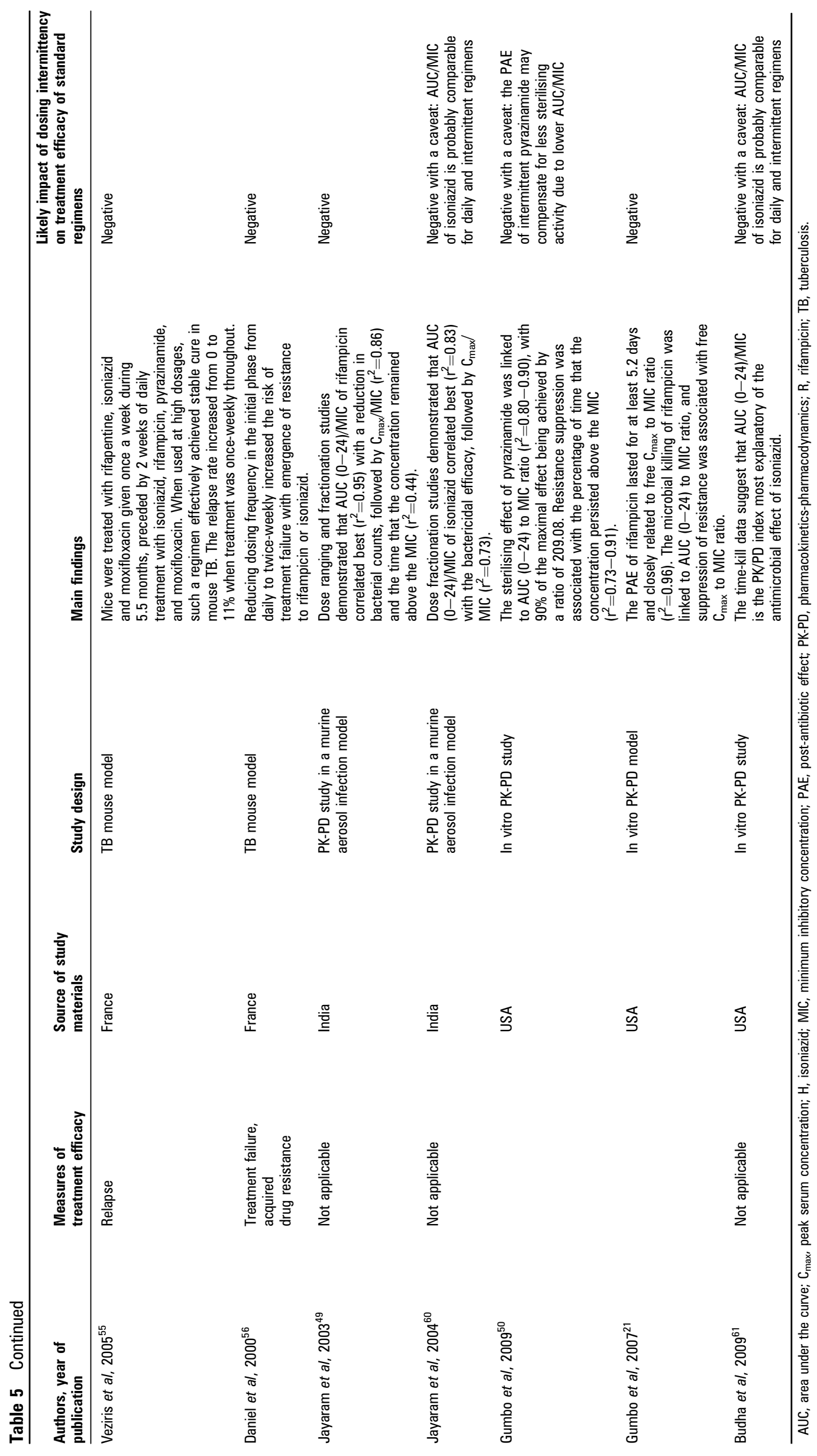


the risk of treatment failure, recurrence and acquired drug resistance (including acquired rifamycin resistance, particularly in patients with advanced HIV infection). Compared with intermittent treatment throughout, daily treatment throughout significantly reduces the risk of relapse in the presence of initial cavitation $^{34} 35$ or HIV infection, ${ }^{32}$ while daily treatment in the initial phase significantly reduces failure, relapse and acquired drug resistance rates among patients with HIV infection ${ }^{31} 33$ or isoniazid-resistant strains. ${ }^{46} 47$ Treatment with a daily initial phase followed by a thrice-weekly continuation phase is probably comparable to daily treatment throughout. ${ }^{35}$ Inconclusive findings from systematic reviews ${ }^{37-40}$ are largely due to insufficient head-to-head comparison of different schedules in controlled clinical trials, which has been provided by only one randomised controlled trial. $^{62} 63$ Negative findings from a systematic review and meta-analysis ${ }^{41}$ may be partly attributable to lack of controlling for major confounders of relapse such as initial cavitation and 2-month sputum culture status, ${ }^{34} 6465$ and partly due to the inclusion of daily regimens containing no rifampicin in the continuation phase, such as isoniazid and ethambutol ${ }^{66}$ or isoniazid and pyrazinamide. ${ }^{67-69}$

The available evidence suggests that the effect of dosing schedules on treatment efficacy is best harnessed in the initial phase rather than in the continuation phase. In fact, there has been in vitro evidence for a few decades that the more rapid the antibacterial effect, the less likely is the emergence of persisters and the lower is the risk of relapse. ${ }^{70}$ It has been hypothesised that there are four subpopulations of tubercle bacilli: actively dividing bacteria, bacteria that divide slowly in an acidic microenvironment, semi-dormant persisters that are metabolically active in spurts, and dormant bacilli. ${ }^{22}$ While rifampicin and pyrazinamide have appreciable sterilising activity, ${ }^{71} 72$ rifampicin is the only first-line TB drug with putative activity against persisters, which are also characterised by phenotypic resistance to isoniazid. This hypothesis of mycobacterial subpopulations has been supplemented by a Yin-Yang model of reverters and persisters, which suggests a dynamic change between bacillary persisters and the other subpopulations during treatment of both TB disease and latent TB infection. ${ }^{5}$ Thus, optimising bactericidal and sterilising effects of TB drugs in the initial phase can minimise the overall bacterial load from which persisters emerged. A smaller population of persisters, which are phenotypically resistant to isoniazid and virtually eradicated only by rifampicin, leads to a lower probability of selecting out rifampicin-resistant mutants (and hence acquired rifamycin resistance) that are inadequately contained by poor host immunity in advanced HIV infection. ${ }^{73}$

Several biological factors may explain why using daily dosing schedules of standard rifampicin regimens improves treatment efficacy. First, while it may be sufficient for drugs with prolonged PAEs to kill rapidly or slowly dividing bacteria that replicate periodically, intermittent treatment may be less efficacious against persisters with intermittent metabolic activity when dosing is asynchronous with the metabolic bursts. Increasing the frequency of dosing reduces the chance of asynchrony. Second, food-drug interaction with rifampicin may cause erratic absorption of rifampicin. ${ }^{74}$ Lastly, protein binding may also reduce penetration of rifampicin into cavities, especially during the continuation phase when there is less inflammation and more fibrosis. ${ }^{75}$ The maximum serum concentration of rifampicin is reduced to approximately $2 \mathrm{mg} / 1$ after food. ${ }^{74}$ It has been shown that broth-determined MIC for rifampicin ranged from 0.06 to $0.25 \mathrm{mg} / \mathrm{l}^{76}$ Assuming that $80 \%$ of rifampicin in blood is bound to protein, ${ }^{74}$ total plasma rifampicin levels of $0.3-1.25 \mathrm{mg} / \mathrm{l}$ can achieve broth-determined MIC. However, based on a peak serum rifampicin level of approximately $12 \mathrm{mg} / \mathrm{l}$ in patients treated with rifampicin $600 \mathrm{mg}$ daily $^{74}$ and a peak sputum rifampicin level of approximately $6 \mathrm{mg} / \mathrm{l}$ at the same rifampicin dosage, ${ }^{74} 78$ rifampicin levels in TB cavities may be about half of serum levels. More frequent dosing may compensate for less sterilising activity and shorter PAEs due to lower rifampicin levels in TB cavities.

It is perhaps important not to forget PAEs in the pursuit of optimal dosing schedules. Twice-weekly high-dose isoniazid is at least as efficacious as daily isoniazid. ${ }^{24}$ Pyrazinamide $3 \mathrm{~g}$ thrice weekly, which is higher than the average dosage used in intermittent regimens, is more effective than $1.5 \mathrm{~g}$ once daily. ${ }^{79}$ In a relatively small clinical trial involving thrice-weekly treatment of patients with predominantly isoniazid-resistant TB with rifampicin, ethambutol and pyrazinamide, the 2-year relapse rate was non-significantly lower among subjects given pyrazinamide $2.5-3 \mathrm{~g}$ thrice weekly than those given pyrazinamide $1.5-2 \mathrm{~g}$ thrice weekly $(11.3 \%$ vs $16.3 \%) .^{80}$ If not for the higher risk of immune-mediated adverse events due to intermittent high-dose rifampicin ${ }^{81-83}$ and unwarranted fear of hepatotoxicity due to intermittent high-dose pyrazinamide, ${ }^{79} 8485$ the observed difference between daily and intermittent treatment regimens might be reduced by increasing dosages of rifampicin and pyrazinamide in intermittent regimens. The much longer elimination half-life of rifapentine may allow intermittent treatment without compromising treatment efficacy, ${ }^{52-54}$ and make it possible to harness PAEs to facilitate DOT and better suppress drug resistance. ${ }^{21}$

Optimising dosing schedules should not be the only approach for improving TB treatment. Lessons from the study of pyrazinamide, which has completely different mechanisms of sterilising activity from rifampicin, have suggested that shortening TB treatment necessitates development of new drugs that are able to eradicate persisters with different modes of action. ${ }^{86}$ Unfortunately, the development of new drugs with good sterilising activity is difficult and in part hampered by the lack of good surrogate markers of relapse. Further studies for identifying better surrogates of relapse seem warranted. ${ }^{87} 88$ In addition, timely initiation of effective antiretroviral treatment in HIV-related TB can restore CD4 counts and reduce the risk of recurrence ${ }^{89}$ and possibly acquired rifamycin resistance.

In conclusion, the current review suggests high levels of evidence for using daily schedules in standard TB treatment regimens, especially during the initial phase in the presence of cavitation, isoniazid resistance and advanced HIV co-infection. It corroborates prevailing understanding of pharmacokineticspharmacodynamics and mycobacterial persisters and supports exploration of rifapentine-containing regimens in higher dosages and frequency. ${ }^{34-45}$

\section{Competing interests None.}

Provenance and peer review Not commissioned; externally peer reviewed.

\section{REFERENCES}

1. Mitchison DA. The search for new sterilizing anti-tuberculosis drugs. Front Biosci 2004:9:1059-72

2. National Tuberculosis Institute. Tuberculosis in a rural population of South India: a five-year epidemiological study. Bull World Health Organ 1974;51:473-88

3. Springett VH. Ten-year results during the introduction of chemotherapy for tuberculosis. Tubercle 1971;52:73-87.

4. Mitchison DA. Role of individual drugs in the chemotherapy of tuberculosis. Int $J$ Tuberc Lung Dis 2000;4:796-806. 
5. Zhang Y. Advances in the treatment of tuberculosis. Clin Pharmacol Ther 2007:82:595-600.

6. Fox W. Self-administration of medicaments. A review of published work and a study of the problems. Bull Int Union Tuberc 1962:32:307-31.

7. Dawson JJ, Devadatta S, Fox W, et al. A 5-year study of patients with pulmonary tuberculosis in a concurrent comparison of home and sanatorium treatment for one year with isoniazid plus PAS. Bull World Health Organ 1966;34:533-51.

8. Kamat SR, Dawson JJ, Devadatta S, et al. A controlled study of the influence of segregation of tuberculous patients for one year on the attack rate of tuberculosis in a 5 -year period in close family contacts in South India. Bull World Health Organ 1966;34:517-32.

9. Fox W, Ellard GA, Mitchison DA. Studies on the treatment of tuberculosis undertaken by the British Medical Research Council tuberculosis units, 1946-1986, with relevant subsequent publications. Int J Tuberc Lung Dis 1999;3:S231-79.

10. World Health Organization. Global Tuberculosis Programme. Global Tuberculosis Control. Geneva, Switzerland: WHO Report, 1997. WHO/GTB/97.225.

11. Kamolratanakul P, Sawert $H$, Lertmaharit $S$, et al. Randomized controlled trial of directly observed treatment (DOT) for patients with pulmonary tuberculosis in Thailand. Trans R Soc Trop Med Hyg 1999;93:552-7.

12. Walley JD, Khan MA, Newell JN, et al. Effectiveness of the direct observation component of DOTS for tuberculosis: a randomised controlled trial in Pakistan. Lancet 2001;357:664-9.

13. Zwarenstein M, Schoeman JH, Vundule $\mathbf{C}$, et al. Randomised controlled trial of selfsupervised and directly observed treatment of tuberculosis. Lancet 1998;352:1340-3.

14. Chaulk CP, Moore-Rice K, Rizzo R, et al. Eleven years of community-based directly observed therapy for tuberculosis. JAMA 1995;274:945-51.

15. Iseman MD, Cohn DL, Sbarbaro JA. Directly observed treatment of tuberculosis We can't afford not to try it. N Engl J Med 1993;328:576-8.

16. Sharma SK, Liu JJ. Progress of DOTS in global tuberculosis control. Lancet 2006;367:951-2.

17. Bayer R, Wilkinson D. Directly observed therapy for tuberculosis: history of an idea. Lancet 1995; 345:1545-8.

18. Chan CY, Au-Yeang C, Yew WW, et al. Postantibiotic effects of antituberculosis agents alone and in combination. Antimicrob Agents Chemother 2001:45:3631-4.

19. Dickinson JM, Mitchison DA. In vitro and in vivo studies to assess the suitability of antituberculous drugs for use in intermittent chemotherapy regimens. Tubercle 1968;49(Suppl):66-70

20. Frieden $\mathbf{T}$. What is the intermittent treatment and what is the scientific basis for intermittency? In: Frieden T, ed. Toman's Tuberculosis. Case Detection Treatment, and Monitoring-Questions and Answers. 2nd edn. Geneva: World Health Organization, 2004:130-8.

21. Gumbo T, Louie A, Deziel MR, et al. Concentration-dependent mycobacterium tuberculosis killing and prevention of resistance by rifampin. Antimicrob Agents Chemother 2007:51:3781-8.

22. Mitchison DA. Basic mechanisms of chemotherapy. Chest 1979:76:771-81.

23. Grumbach F, Canetti G, Grosset J, et al. Late results of long-term intermittent chemotherapy of advanced, murine tuberculosis: limits of the murine model. Tubercle 1967:48:11-26.

24. Lotte A, Hatton F, Perdrizet S, et al. A concurrent comparison of intermittent (twice-weekly) isoniazid plus streptomycin and daily isoniazid plus PAS in the domiciliary treatment of pulmonary tuberculosis; tuberculosis chemotherapy centre, Madras. Bull World Health Organ 1964;31:247-71.

25. Cao JP, Zhang LY, Zhu JQ, et al. Two-year follow-up of directly-observed intermittent regimens for smear-positive pulmonary tuberculosis in China. Int $\mathrm{J}$ Tuberc Lung Dis 1998;2:360-4.

26. Chaisson RE, Clermont HC, Holt EA, et al. Six-month supervised intermittent tuberculosis therapy in Haitian patients with and without HIV infection. Am J Respir Crit Care Med 1996:154:1034-8.

27. Zhanel GG, Hoban DJ, Harding GK. The postantibiotic effect: a review of in vitro and in vivo data. DICP 1991;25:153-63.

28. Frieden T ed Toman's Tuberculosis. Case Detection Treatment and Monitoring Questions and Answers. 2nd edn. Geneva: World Health Organization, 2004.

29. Scottish Intercollegiate Guidelines Network. SIGN 50: A Guideline Developer's Handbook. Edinburgh: SIGN:2008.

30. Anon. Low rate of emergence of drug resistance in sputum positive patients treated with short course chemotherapy. Int J Tuberc Lung Dis 2001:5:40-5.

31. Khan FA, Minion J, Pai M, et al. Treatment of active tuberculosis in HIV-coinfected patients: a systematic review and meta-analysis. Clin Infect Dis 2010;50:1288-99.

32. Nahid P, Gonzalez LC, Rudoy I, et al. Treatment outcomes of patients with HIV and tuberculosis. Am J Respir Crit Care Med 2007:175:1199-206.

33. Li J, Munsiff SS, Driver CR, et al. Relapse and acquired rifampin resistance in HIVinfected patients with tuberculosis treated with rifampin- or rifabutin-based regimens in New York City, 1997-2000. Clin Infect Dis 2005;41:83-91.

34. Chang KC, Leung CC, Yew WW, et al. A nested case-control study on treatmentrelated risk factors for early relapse of tuberculosis. Am J Respir Crit Care Med 2004;170:1124-30.

35. Chang KC, Leung CC, Yew WW, et al. Dosing schedules of 6-month regimens and relapse for pulmonary tuberculosis. Am J Respir Crit Care Med 2006:174:1153-8.

36. Gao X, Li J, Yang Z, et al. Rifapentine vs rifampicin for the treatment of pulmonary tuberculosis: a systematic review. Int J Tuberc Lung Dis 2009:13:810-19.

37. Mwandumba HC, Squire SB. Fully intermittent dosing with drugs for tuberculosis. Cochrane Database Syst Rev 2000:CD000970.
38. Mwandumba HC, Squire SB. Fully intermittent dosing with drugs for treating tuberculosis in adults. Cochrane Database Syst Rev 2001:CD000970.

39. Cox HS, Morrow M, Deutschmann PW. Long term efficacy of DOTS regimens for tuberculosis: systematic review. BMJ 2008;336:484-7.

40. Ziganshina LE, Garner P. Tuberculosis (HIV-negative people). Clin Evid (Online) 2009:4:904.

41. Menzies D, Benedetti A, Paydar A, et al. Effect of duration and intermittency of rifampin on tuberculosis treatment outcomes: a systematic review and metaanalysis. PLoS Med 2009;6:e1000146.

42. Prasad R, Garg R, Mukerji P, et al. Comparative study of daily and thrice weekly intermittent regimens given for six months in the treatment of pulmonary tuberculosis. J Intern Med India 2001:4:1-3.

43. Alvarez TA, Rodrigues MP, Viegas CA. Prevalence of drug-resistant mycobacterium tuberculosis in patients under intermittent or daily treatment. J Bras Pneumol 2009:35:555-60.

44. Paramasivan CN, Chandrasekaran V, Santha T, et al. Bacteriological investigations for short-course chemotherapy under the tuberculosis programme in two districts of India. Tuberc Lung Dis 1993;74:23-7.

45. Singla R, Srinath D, Gupta $S$, et al. Risk factors for new pulmonary tuberculosis patients failing treatment under the revised National Tuberculosis Control Programme, India. Int J Tuberc Lung Dis 2009;13:521-6.

46. Menzies D, Benedetti A, Paydar A, et al. Standardized treatment of active tuberculosis in patients with previous treatment and/or with mono-resistance to isoniazid: a systematic review and meta-analysis. PLoS Med 2009;6:e1000150.

47. Escalante $\mathbf{P}$, Graviss $\mathrm{EA}$, Griffith $\mathrm{DE}$, et al. Treatment of isoniazid-resistant tuberculosis in southeastern Texas. Chest 2001:119:1730-6.

48. Menon PR, Lodha R, Sivanandan S, et al. Intermittent or daily short course chemotherapy for tuberculosis in children: meta-analysis of randomized controlled trials. Indian Pediatr 2010;47:67-73.

49. Jayaram R, Gaonkar S, Kaur P, et al. Pharmacokinetics-pharmacodynamics of rifampin in an aerosol infection model of tuberculosis. Antimicrob Agents Chemother 2003;47:2118-24

50. Gumbo T, Dona CSWS, Meek C, et al. Pharmacokinetics-pharmacodynamics of pyrazinamide in a novel in vitro model of tuberculosis for sterilizing effect: a paradigm for faster assessment of new antituberculosis drugs. Antimicrob Agents Chemother 2009:53:3197-204

51. Weiner $\mathbf{M}$, Benator D, Burman W, et al. Association between acquired rifamycin resistance and the pharmacokinetics of rifabutin and isoniazid among patients with HIV and tuberculosis. Clin Infect Dis 2005:40:1481-91.

52. Rosenthal IM, Zhang M, Almeida D, et al. Isoniazid or moxifloxacin in rifapentinebased regimens for experimental tuberculosis? Am J Respir Crit Care Med 2008:178:989-93.

53. Rosenthal IM, Zhang M, Williams KN, et al. Daily dosing of rifapentine cures tuberculosis in three months or less in the murine model. PLoS Med 2007;4:e344.

54. Rosenthal IM, Williams K, Tyagi S, et al. Potent twice-weekly rifapentine-containing regimens in murine tuberculosis. Am J Respir Crit Care Med 2006;174:94-101.

55. Veziris $\mathbf{N}$, Lounis $N$, Chauffour A, et al. Efficient intermittent rifapentinemoxifloxacin-containing short-course regimen for treatment of tuberculosis in mice. Antimicrob Agents Chemother 2005;49:4015-19.

56. Daniel N, Lounis N, Ji B, et al. Antituberculosis activity of once-weekly rifapentine-containing regimens in mice. Long-term effectiveness with 6- and 8-month treatment regimens. Am J Respir Crit Care Med 2000;161:1572-7.

57. Weiner M, Burman W, Vernon A, et al. Low isoniazid concentrations and outcome of tuberculosis treatment with once-weekly isoniazid and rifapentine. Am J Respir Crit Care Med 2003;167:1341-7.

58. Goutelle S, Bourguignon L, Maire PH, et al. Population modeling and Monte Carlo simulation study of the pharmacokinetics and antituberculosis pharmacodynamics of rifampin in lungs. Antimicrob Agents Chemother 2009:53:2974-81.

59. Gupta P, Roy V, Sethi GR, et al. Pyrazinamide blood concentrations in children suffering from tuberculosis: a comparative study at two doses. Br J Clin Pharmacol 2008:65:423-7.

60. Jayaram R, Shandil RK, Gaonkar S, et al. Isoniazid pharmacokineticspharmacodynamics in an aerosol infection model of tuberculosis. Antimicrob Agents Chemother 2004:48:2951-7.

61. Budha NR, Lee RB, Hurdle JG, et al. A simple in vitro PK/PD model system to determine time-kill curves of drugs against Mycobacteria. Tuberculosis (Edinb) 2009;89:378-85.

62. Hong Kong Chest Service/British Medical Research Council. Controlled trial of four thrice-weekly regimens and a daily regimen all given for 6 months for pulmonary tuberculosis. Lancet 1981;1:171-4.

63. Hong Kong Chest Service/British Medical Research Council. Controlled trial of 4 three-times-weekly regimens and a daily regimen all given for 6 months for pulmonary tuberculosis. Second report: the results up to 24 months. Hong Kong Chest Service/British Medical Research Council. Tubercle 1982;63 89-98.

64. Benator D, Bhattacharya M, Bozeman L, et al. Rifapentine and isoniazid once a week versus rifampicin and isoniazid twice a week for treatment of drugsusceptible pulmonary tuberculosis in HIV-negative patients: a randomised clinical trial. Lancet 2002;360:528-34

65. Zierski M, Bek E, Long MW, et al. Short-course (6-month) cooperative tuberculosis study in Poland: results 18 months after completion of treatment. Am Rev Respir Dis 1980;122:879-89. 
66. Jindani A, Nunn AJ, Enarson DA. Two 8-month regimens of chemotherapy for treatment of newly diagnosed pulmonary tuberculosis: international multicentre randomised trial. Lancet 2004;364:1244-51.

67. Anon. Controlled clinical trial of five short-course (4-month) chemotherapy regimens in pulmonary tuberculosis. First report of 4th study. East African and British Medical Research Councils. Lancet 1978;2:334-8.

68. Anon. Controlled clinical trial of 4 short-couse regimens of chemotherapy (three 6month and one 8-month) for pulmonary tuberculosis. Tubercle 1983;64:153-66

69. Anon. Controlled clinical trial of 4 short-course regimens of chemotherapy (three 6-month and one 8-month) for pulmonary tuberculosis: final report. East and Central African/British Medical Research Council Fifth Collaborative Study. Tubercle 1986;67:5-15.

70. Crofton J, Douglas E. Respiratory Diseases. 2nd edn. Oxford: Blackwell Scientific, 1975.

71. East African/British Medical Research Councils. Controlled clinical trial of four short-course (6-month) regimens of chemotherapy for treatment of pulmonary tuberculosis. Second report. Lancet 1973;1:1331-8.

72. Mitchison DA, Nunn AJ. Influence of initial drug resistance on the response to short-course chemotherapy of pulmonary tuberculosis. Am Rev Respir Dis 1986;133:423-30.

73. Mitchison DA. How drug resistance emerges as a result of poor compliance during short course chemotherapy for tuberculosis. Int J Tuberc Lung Dis 1998;2:10-5.

74. Acocella G. Clinical pharmacokinetics of rifampicin. Clin Pharmacokinet 1978;3:108-27.

75. Mitchison DA. Role of isoniazid in once-weekly rifapentine treatment of pulmonary tuberculosis. Am J Respir Crit Care Med 2003;167:1298-9.

76. Heifets L. Qualitative and quantitative drug-susceptibility tests in mycobacteriology. Am Rev Respir Dis 1988;137:1217-22.

77. Acocella G, Pagani V, Marchetti M, et al. Kinetic studies on rifampicin. I. Serum concentration analysis in subjects treated with different oral doses over a period of two weeks. Chemotherapy 1971;16:356-70.
78. Binda G, Domenichini E, Gottardi A et al. Rifampicin, a general review. Arzneimittelforschung 1971;21:1907-77.

79. East African/British Medical Research Council Pyrazinamide Investigation. A controlled comparison of four regimens of streptomycin plus pyrazinamide in the retreatment of pulmonary tuberculosis. Tubercle 1969;50:81-114.

80. Hong YP, Kim SC, Chang SC, et al. Comparison of a daily and three intermittent retreatment regimens for pulmonary tuberculosis administered under programme conditions. Tubercle 1988;69:241-53.

81. Dickinson JM, Mitchison DA, Lee SK, et al Serum rifampicin concentration related to dose size and to the incidence of the 'flu' syndrome during intermittent rifampicin administration. J Antimicrob Chemother 1977;3:445-52.

82. Girling DJ. Hong Kong Treatment Services-Royal Postgraduate Medical SchoolBritish Medical Research Council Co-operative study of rifampicin plus ethambutol in daily and intermittent regimens. Clinical observations on adverse reactions. Scand J Respir Dis Supp/ 1973:84:119-24.

83. Peloquin C. What is the 'right' dose of rifampin? Int J Tuberc Lung Dis 2003;7:3-5

84. Ramakrishnan CV, Janardhanam B, Krishnamurthy DV, et al. Toxicity of pyrazinamide, administered once weekly in high dosage, in tuberculous patients. Bull World Health Organ 1968;39:775-9.

85. Kamal N, Mukerjee PK, Kishore K, et al. Liver functions during pyrazinamide therapy. Indian J Tuberc 1976;23:14-18.

86. Zhang $\mathbf{Y}$, Mitchison $\mathrm{D}$. The curious characteristics of pyrazinamide: a review. Int $J$ Tuberc Lung Dis 2003:7:6-21.

87. Burman WJ. The hunt for the elusive surrogate marker of sterilizing activity in tuberculosis treatment. Am J Respir Crit Care Med 2003;167:1299-301.

88. Horne DJ, Royce SE, Gooze L, et al. Sputum monitoring during tuberculosis treatment for predicting outcome: systematic review and meta-analysis. Lancet Infect Dis 2010;10:387-94.

89. Golub JE, Durovni B, King BS, et al. Recurrent tuberculosis in HIV-infected patients in Rio de Janeiro, Brazil. AIDS 2008;22:2527-33.

\section{Scadding-Morriston Davies joint fellowship in respiratory medicine 2012}

This fellowship is available to support visits to medical centres in U.K. or abroad for the purpose of undertaking studies related to respiratory medicine. Applications are invited from medical graduates practising in the United Kingdom, including consultants and irrespective of the number of years in that grade. There is no application form but a curriculum vitae should be submitted together with a detailed account of the duration and nature of the work and the centres to be visited, confirming that these have agreed to provide the facilities required. Please state the sum of money needed for travel and subsistence. A sum of up to $£ 20,000$ can be awarded to the successful candidate, or the sum may be divided to support two or more applications. Applications should be sent to the Secretary by 31 January 2012.

Dr I A Campbell

Secretary to the Scadding-Morriston Davies Fellowship

Department of Respiratory Medicine

Academic Centre $\left(2^{\text {nd }}\right.$ Floor $)$

Llandough Hospital

PENARTH, Vale of Glamorgan

CF64 2XX

lan.campbell@wales.nhs.uk 